\title{
Pharmacokinetic evaluation of atorvastatin and sitagliptin in combination for the treatment of type 2 diabetes
}

\author{
André J Scheen
}

University of Liège, Division of Diabetes, Nutrition and Metabolic Disorders and Division of Clinical Pharmacology, Department of Medicine, CHU Sart Tilman, Liège, Belgium

\section{Abstract}

Introduction: Patients with type 2 diabetes (T2DM) are exposed to a high risk of cardiovascular disease (CVD) requiring a global therapeutic approach. Statin therapy has proven its efficacy in reducing CVD events in T2DM patients. Dipeptidylpeptidase-4 inhibitors (gliptins), which are increasingly used to target hyperglycemia, also offer promising preliminary results regarding a possible reduction in CVD events. As most patients with T2DM may be treated with both a statin and a gliptin, dual pharmacological therapy, possibly as a fixed-dose combination (FDC), deserves further consideration.

Areas covered: The reader is provided with an update of information on the pharmacokinetics (PK) and pharmacodynamics (PD) of atorvastatin and sitagliptin. The article provides an analysis of the potential PK/PD interactions between the two compounds and puts into perspective the potential cardiovascular protection that such a dual therapy may offer in patients with T2DM.

Expert opinion: Atorvastatin and sitagliptin are not prone to PK drug-drug interactions. Their coadministration, either separately or in an FDC, improves both blood glucose levels and cholesterol concentrations, without clinically relevant adverse events. The atorvastatin plus sitagliptin combination may be used to reduce LDL cholesterol and hyperglycemia in patients with T2DM, with the aim to improve CVD prognosis and adherence to therapy.

Keywords : atorvastatin ; cardiovascular disease ; DPP-4 inhibitor ; pharmacokinetics ; sitagliptin ; type 2 diabetes mellitus

Box 1. Drug summary.

Drug name

Phase

Indication

Pharmacology description

Route administration Chemical structure
Atorvastatin/sitagliptin

Available separately

Fixed-dose combination in Phase III

Cardiovascular protection in patients with T2DM

Atorvastatin: HMG-COA reductase inhibitor improving lipid profile Sitagliptin: DPP-4 inhibitor improving glucose control Oral<smiles>N=C(CC(=O)N1CCn2c(nnc2P(F)(F)(F)F)C1)Cc1cc(F)c(F)cc1F</smiles><smiles>Cc1ccc(-c2c(-c3ccccc3)c(C(=O)Nc3ccccc3)c(C(C)C)n2CC[C@@H](O)C[C@@H](O)CC(=O)O)cc1</smiles>

$[9,63,65]$ 


\section{Article highlights}

- Patients with type 2 diabetes mellitus (T2DM) are exposed to a high risk of cardiovascular disease (CVD) and most of them should receive a statin irrespective of their lipid profile.

- In CARDS, but not in ASPEN, atorvastatin has proven its remarkable efficacy in improving lipid profile and reducing CVD events in patients with T2DM. Nevertheless, some recent data suggested that atorvastatin, like other statins, may slightly deteriorate glucose control and increase new-onset diabetes. - DPP-4 inhibitors (gliptins) are new incretin-based oral glucose-lowering agents that improve fasting and postprandial glucose levels (thus resulting in significant reduction in $\mathrm{HbA} 1 \mathrm{c}$ ), without promoting hypoglycemia or weight gain (two adverse events that may increase the CVD risk).

- Sitagliptin, the first in class among DPP-4 inhibitors, slightly improves lipid profile (especially postprandially), besides its glucose-lowering activity, and may also exert other positive effects on cardiovascular system via increased GLP-1 levels.

- The encouraging results obtained in Phase III program with less CVD events with sitagliptin than with comparators (placebo or active) triggers the large prospective cardiovascular outcome trial TECOS, in which numerous T2DM patients will probably be treated simultaneously with sitagliptin and atorvastatin (or another statin).

- Combination of atorvastatin and sitagliptin may provide an additive protective cardiovascular effect, although this has not been specifically proven yet.

- A fixed-dose combination (FDC) combining atorvastatin and sitagliptin would reduce the number of pills and possibly improve adherence, although a careful PK study testing such an FDC is not available yet. On note, sitagliptin requires dose reduction in case of renal impairment, but not atorvastatin.

- The place of an atorvastatin-sitagliptin FDC should be better evaluated for the management of patients with T2DM in clinical practice, because two other FDCs are already available, sitagliptin-metformin and amlodipine-atorvastatin.

This box summarizes key points contained in the article.

\section{Introduction}

Patients with type 2 diabetes mellitus (T2DM) are exposed to a high coronary risk. It is generally considered that almost two-thirds of patients will die from cardiovascular disease (CVD) and 50\% from myocardial infarct or related complications. Thereby primary prevention of CVD in people with T2DM is a key objective as emphasized in both European and US guidelines [1,2]. Lipid-lowering therapy plays a major role in the prevention of CVD, and statin is the pharmacological class that has proven the best efficacy in both primary and secondary preventions [3]. Thus, according to recent guidelines from the American Diabetes Association [4] and the European Society of Cardiology and the European Atherosclerosis Society [5], most (if not all) T2DM patients must receive a statin therapy. This recommendation is supported by the significant $21 \%$ proportional reduction in major vascular events per mmol/l reduction in LDL cholesterol provided by statin therapy in patients with T2DM [6], a figure similar to that observed in the general population [3].

Atorvastatin is one of the most potent and extensively investigated hydroxymethylglutaryl coenzyme A (HMGCoA) reductase inhibitor. It lowers plasma total and LDL (low-density lipoprotein) cholesterol levels by inhibiting endogenous cholesterol synthesis and increasing the amount of available LDL receptors in hepatocyte membranes; it also reduces triglyceride (TG) levels, through an as yet unproven mechanism, which may offer an add-on value to treat diabetic dyslipidemia [7,8]. In the Collaborative Atorvastatin Diabetes Study (CARDS), atorvastatin $10 \mathrm{mg} /$ day reduced the risk of first CVD events by almost one-third in patients with T2DM without high LDL cholesterol [9]. However, in the Atorvastatin Study for Prevention of Coronary Heart Disease Endpoints (ASPEN) in non-insulin-dependent diabetes mellitus, only a 10\% (nonsignificant) reduction in composite primary cardiovascular endpoint was reported in the group receiving atorvastatin $10 \mathrm{mg}$, a finding that may somewhat attenuate the overall protective effect that may be expected from atorvastatin in patients with T2DM [10].

Although the relationship is less strong than that with LDL cholesterol, there is a relationship between CVD risk and levels of glycemia. A collaborative meta-analysis of 102 prospective studies showed that diabetes confers about a twofold excess risk for a wide range of vascular diseases, independently from other conventional risk factors. In people without diabetes, fasting blood glucose concentration is modestly and nonlinearly associated with the risk of CVD [11]. Besides fasting glycemia, most epidemiological data also implicate postprandial hyperglycemia in the development of CVD, suggesting that glucose-lowering agents should also tackle postmeal hyperglycemia [12]. In addition, the confounding role of hypoglycemia has been pointed out [13]. The controversy has been magnified since the publication of the ACCORD (Action to Control Cardiovascular Risk in 
Diabetes) data suggesting a significant relationship between severe symptomatic [14] and even frequent and unrecognized hypoglycemia [15], on the one hand, and increased CVD mortality, on the other hand. Therefore, the use of glucose-lowering agents that reduce both fasting and postprandial hyperglycemia without increasing the risk of hypoglycemia, such as inhibitors of dipeptidyl peptidase-4 (DPP-4) (also called gliptins) [16], may offer clinically relevant advantages over sulfonylureas [17]. Indeed, the risk of (severe) hypoglycemia linked to sulfonylureas is well known [18]. In addition, from a pharmacokinetic point of view, sulfonylureas are exposed to drug-drug interactions (DDIs) [19], which is not the case for most DPP-4 inhibitors [20]. This property may offer additional clinical benefit because patients with T2DM, especially in presence of CVD, are commonly treated by numerous medications and thus confronted to many potential DDIs [21].

Sitagliptin (Januvia) has been the first DPP-4 inhibitor launched worldwide and benefits from the largest clinical experience [22]. It has shown its ability to improve glycemic control in T2DM patients treated by diet alone, metformin, sulfonylurea, glitazone, a metformin-sufonylurea combined therapy or even insulin [23]. As metformin is considered as the first-line medication in the management of T2DM, the most popular association is sitagliptin added to metformin, a combination that is available as fixed-dose combination (FDC) and largely used in clinical practice [24,25].

Most patients with T2DM should take several medications every day and compliance to drug therapy is increasingly viewed as a major concern [26-28]. The use of FDC may offer the advantage of reducing the number of tablets to be taken and thereby increase patient's adherence to therapy [29]. Sitagliptin is available worldwide in combination with metformin $[24,25]$. Of note, atorvastatin was successfully combined with amlodipine, considering that hypertension and hypercholesterolemia are commonly associated and that both represent well-known CVD risk factors [30]. Because a statin is recommended in most patients with T2DM, another FDC might be considered in the future with the association of atorvastatin and sitagliptin. The present paper provides a pharmacokinetic (PK) and pharmacodynamic (PD) evaluation of atorvastatin and sitagliptin (see (Box 1)), either separately or combination. To identify relevant studies, an extensive literature search of MEDLINE was performed from January 2006 to March 2012, with the two key words 'atorvastatin' and 'sitagliptin.' No language restrictions were imposed. Reference lists of original studies, narrative reviews and previous systematic reviews were also examined.

\section{Pharmacokinetic evaluation}

\subsection{Atorvastatin}

Atorvastatin acid is highly soluble and permeable, and the drug is completely absorbed after oral administration. However, atorvastatin is subject to extensive first-pass metabolism in the gut wall as well as in the liver. As a consequence, oral bioavailability is rather low, around 14\%. Food decreases the absorption rate of atorvastatin after oral administration, as indicated by decreased peak concentration and increased time to peak concentration [31].

The volume of distribution of atorvastatin is 3811 , and plasma protein binding exceeds $98 \%$. The total plasma clearance of atorvastatin is $625 \mathrm{ml} / \mathrm{min}$ and the half-life is about $7 \mathrm{~h}$. The renal route is of minor importance $(<1 \%)$ for the elimination of atorvastatin. Atorvastatin is extensively metabolized in both the gut and liver by oxidation, lactonization and glucuronidation, and the metabolites are eliminated by biliary excretion and direct secretion from blood to the intestine. In vitro, atorvastatin is a substrate for P-glycoprotein (Pgp), organic aniontransporting polypeptide (OATP) $\mathrm{C}$ and $\mathrm{H}+-$ monocarboxylic acid cotransporter. In vivo, cytochrome $\mathrm{P} 450$ (CYP) 3A4 is responsible for the formation of two active metabolites from the acid and the lactone forms of atorvastatin. Finally, atorvastatin and its metabolites undergo glucuronidation [31].

Because atorvastatin is subject to metabolism by CYP3A4 and cellular membrane transport by OATP C and Pgp, DDIs with potent inhibitors of these systems, such as itraconazole, nelfinavir, ritonavir, ciclosporin, fibrates, erythromycin and grapefruit juice, have been demonstrated [21]. An interaction with gemfibrozil seems to be mediated by inhibition of glucuronidation. A few case studies have reported rhabdomyolysis when the PK of atorvastatin has been affected by interacting drugs [32]. Atorvastatin increases the bioavailability of digoxin, most probably by inhibition of Pgp, but does not affect the PK of ritonavir or nelfinavir [31].

\subsection{Sitagliptin}

We extensively described the PK properties of sitagliptin [33] and well as its low potential for DDIs [20]. 
To determine the absolute bioavailability of sitagliptin and the effect of a high fat meal on sitagliptin PK, healthy subjects were randomized to each of three open-label treatments: an intravenous $100 \mathrm{mg}$ dose of sitagliptin; a single oral $100 \mathrm{mg}$ tablet of sitagliptin administered following a high fat meal; and a single oral $100 \mathrm{mg}$ sitagliptin administered fasted [34]. The mean absolute bioavailability of sitagliptin was $87 \%$ with a $90 \%$ confidence interval (CI) of $(81 \%, 93 \%)$. Interestingly, food did not affect sitagliptin PK and, therefore, the drug can be administered without regard to meal.

The metabolism and excretion of ${ }^{14} \mathrm{C}$-sitagliptin were investigated in humans after a single oral dose of 83 $\mathrm{mg} / 193 \mu \mathrm{Ci}$ [35]. Sitagliptin does not appear to undergo extensive metabolism. The primary route of excretion of radioactivity was via the kidneys, with a mean value of $87 \%$ of the administered dose recovered in urine. Mean fecal excretion was $13 \%$ of the administered dose. Parent drug was the major radioactive component in plasma, urine and feces, with only $16 \%$ of the dose excreted as metabolites (13\% in urine and $3 \%$ in feces), indicating that sitagliptin was eliminated primarily by renal excretion. CYP3A4 was the major cytochrome P450 isoenzyme responsible for the limited oxidative metabolism of sitagliptin, with some minor contribution from CYP2C8. However, the contribution of CYP system in sitagliptin metabolism is very small so that clinically relevant DDIs via CYP3A4 or CYP2C8 would probably be negligible [20].

As previously reviewed [33], sitagliptin is excreted into the urine via active tubular secretion and glomerular filtration. Sitagliptin is transported by hOAT3, organic anion transporting polypeptide OATP4C1 and multidrug resistance (MDR) Pgp. In vitro studies suggested that hOAT3, OATP4C1 and MDR1 Pgp might play a role in transporting sitagliptin into and out of renal proximal tubule cells, respectively [36]. However, experimental in vitro data indicated that sitagliptin is unlikely to be a perpetrator of DDIs with Pgp, hOATl or hOAT3 substrates at clinically relevant concentrations and no in vivo clinically meaningful interactions have been described yet [20]. According to in vitro studies, sitagliptin is not an inhibitor of CYP isoenzymes CYP3A4, 2C8, 2C9, 2D6, $1 \mathrm{~A} 2,2 \mathrm{C} 19$ or $2 \mathrm{~B} 6$ and is not an inducer of CYP3A4. Based on these results, sitagliptin is considered unlikely to cause DDIs with other medications that utilize these pathways, and more particularly atorvastatin [20].

Because sitagliptin is essentially excreted via the kidney, a study evaluated the PK of single doses of sitagliptin in patients with various degrees of renal insufficiency (RI) [37]. According to the results of this study, the recommended sitagliptin dosage adjustments are as follows: no adjustment for patients with mild RI (creatinine clearance $50-80 \mathrm{ml} / \mathrm{min}$ ), a twofold decrease in the clinical dose of $100 \mathrm{mg}$ q.d. (i.e., $50 \mathrm{mg}$ q.d.) for patients with moderate RI (creatinine clearance $30-50 \mathrm{ml} / \mathrm{min}$ ) and a fourfold decrease in the clinical dose (25 mg q.d.) for patients with severe RI (creatinine clearance $<30 \mathrm{ml} / \mathrm{min}$ ) or end-stage renal disease.

\subsection{Atorvastatin plus sitagliptin}

In contrast to what has been previously reported with the amlodipine/atorvastatin combination [30] or the metformin/ sitagliptin combination [24,25], no such studies are available yet regarding the atorvastatin plus sitagliptin combination.

As an example, the amlodipine/atorvastatin combination (Caduet ) can be briefly described as it may be considered as a model for a future atorvastatin/sitagliptin combination. It is a single-tablet FDC of the dihydropyridine calcium channel antagonist amlodipine, targeting high blood pressure, and the HMG-CoA reductase inhibitor atorvastatin, targeting hypercholesterolemia. The bioavailability of amlodipine and atorvastatin with the FDC was not significantly different from that with coadministered separate amlodipine and atorvastatin tablets [38]. Amlodipine/atorvastatin offers a convenient and effective approach to improving adherence and managing CV risk in hypertensive patients with dyslipidemia or at risk of CVD [30].

One study in healthy subjects evaluated potential PK interactions between sitagliptin and simvastatin, an HMGCoA reductase inhibitor that shares some PK characteristics with atorvastatin. Coadministration of sitagliptin (100 mg twice daily for 5 days) and simvastatin (20 mg single dose) had no meaningful effect on the PK for active HMG-CoA reductase inhibitors, with a geometric mean ratio (GMR) (90\% CI) for area under the curve of plasma concentrations $\left(\mathrm{AUC}_{0 \text { - last }}\right)$ and maximal concentrations $\left(\mathrm{C}_{\max }\right)$ of $1.06(0.88-1.26)$ and $0.94(0.66-$ 1.34), respectively. There was also no statistically meaningful difference in $\mathrm{T}_{\max }$ between treatments. The conclusion was that steady-state sitagliptin does not alter the PK of a single dose of simvastatin [39]. We might speculate that it should be also the case with atorvastatin, although this remains to be proven.

Finally, a study evaluated the PK interaction of sitagliptin with another lipid-lowering agent. The bioavailability of sitagliptin $100 \mathrm{mg}$ was increased by $54 \%$ when coadministered with gemfibrozil $600 \mathrm{mg}$ twice daily. Even if it was concluded that this interaction may not have any clinical significance as sitagliptin has a wide therapeutic 
index, this conclusion may not hold if the dose of sitagliptin is increased or if is co-prescribed with other antidiabetic drugs and/or CYP 2C8/ human organic anion transporter-3 inhibitors [40]. However, atorvastatin does not share the properties of gemfibrozil, which is a strong inhibitor of CYP2C8 and OATP1B1 [21].

\section{Pharmacodynamic evaluation}

There is no direct PD interaction between atorvastatin, a lipid-lowering agent, and sitagliptin, a glucose-lowering agent. However, besides their primary activity on lipid and glucose metabolism, respectively, some studies have reported that HMG-CoA reductase inhibitors (atorvastatin) may somewhat negatively influence glucose metabolism while others described some positive effects of DPP-4 inhibitors (sitagliptin) on lipid metabolism.

\subsection{Effect of atorvastatin on lipid and glucose metabolism}

\subsubsection{Atorvastatin and lipid profile}

Atorvastatin is a potent synthetic HMG-CoA reductase inhibitor, which explains most of its metabolic and protective activities (Figure 1). In dosages of 10 - $80 \mathrm{mg} /$ day, atorvastatin reduces levels of total cholesterol, LDL cholesterol, TG, very low-density lipoprotein (VLDL) cholesterol and non-high-density lipoprotein (HDL) cholesterol, and increases HDL cholesterol in patients with a wide variety of dyslipidemias. In large long-term trials in patients with primary hypercholesterolemia, atorvastatin produced greater reductions in total cholesterol, LDL cholesterol and TG levels than other HMG-CoA reductase inhibitors (lovastatin, pravastatin, fluvastatin and simvastatin) [8]. However, in subjects with T2DM, smaller improvements of apoB/ apoA1 and across the lipid profile were observed with atorvastatin $(20-80 \mathrm{mg})$ compared with rosuvastatin $(10-40 \mathrm{mg})$ [41]. In the DALI (Diabetes Atorvastatin Lipid Intervention) study, administration of 10 and $80 \mathrm{mg}$ doses of atorvastatin provided similar, significant reductions from baseline in TG levels in patients with T2DM across the dose range, whereas atorvastatin improved cholesterol-related parameters in a dose-dependent manner [42]. In the CARDS study performed in T2DM patients without high cholesterol levels, allocation to atorvastatin $10 \mathrm{mg}$ was associated with a net reduction in LDL cholesterol $(-40 \%)$ and TG $(-19 \%)$ and a negligible increase in HDL cholesterol $(+1 \%)$, changes that were associated with a significant reduction in first CVD events (see below) [9].

\subsubsection{Atorvastatin and glucose control}

In a collaborative meta-analysis of 13 randomized statin trials with 91,140 participants, of whom 4278 (2226 assigned statins and 2052 assigned control treatment) developed diabetes during a mean of 4 years, statin therapy was associated with a modest (9\%) but significant increased risk for incident diabetes (odds ratio (OR) 1.09; 95\% CI 1.02 - 1.17). Treatment of 255 (95\% CI 150 - 852) patients with statins for 4 years resulted in one extra case of diabetes. The conclusion was that statin therapy is associated with a slightly increased risk of development of diabetes, but the risk is low both in absolute terms and when compared with the reduction in coronary events. Clinical practice in patients with moderate or high cardiovascular risk or existing CVD should not change [43]. Although little heterogeneity $(\mathrm{I}(2)=11 \%)$ between trials was noticed in the collaborative metaanalysis [43], potential differences between individual statins have been suggested, with pravastatin promoting risk reduction for new onset of diabetes contrasting with other statins (simvastatin, atorvastatin, rosuvastatin) that all promote significant increase in this risk [44]. These findings may suggest a potential preferable effect of the hydrophilic statins (such as pravastatin) as compared with lipophilic components of the class (atorvastatin, simvastatin) [45]. Indeed differences on insulin sensitivity in non-diabetic subjects between individual statins probably exist that may at least partially explain the findings of previously conducted meta-analyses examining the impact of statins on the development of diabetes [46].

Controversial effects of atorvastatin on glucose control and insulin sensitivity have been reported. A randomized, single-blind, placebo-controlled parallel study was conducted in patients taking placebo and in patients given atorvastatin 10,20, 40 and $80 \mathrm{mg} /$ day, respectively, during a 2-month treatment period. Despite beneficial reductions in LDL cholesterol and apolipoprotein $\mathrm{B}$, atorvastatin treatment resulted in significant increases in fasting insulin and glycated hemoglobin (HbA1c) levels consistent with insulin resistance and increased ambient glycemia in hypercholester-olemic patients [47]. A 24-week, open-label, randomized, parallel-group, Phase IIIb, multi-center study (CORALL) was designed to compare the cholesterol-lowering effects of rosuvastatin compared with atorvastatin in patients with T2DM. Mean fasting plasma glucose increased from baseline 8.7 to $9.5 \mathrm{mmol} / \mathrm{l}$ upon treatment with atorvastatin $20 \mathrm{mg}$ and $9.0 \mathrm{mmol} / 1 \mathrm{after}$ treatment with $80 \mathrm{mg}$. Treatment with the highest dose of atorvastatin $(80 \mathrm{mg}$ ) was associated with mild but significant increase in HbA1c levels from $57 \mathrm{mmol} / \mathrm{l}(7.4 \%)$ at baseline to $61 \mathrm{mmol} / \mathrm{mol}(7.7 \%)$. Future controlled studies are needed to verify these findings and, if confirmed, determine whether such changes represent a true decline in glycemic control [48]. 
High-dose $(80 \mathrm{mg}$ ) atorvastatin treatment compared with placebo in the SPARCL (Stroke Prevention by Aggressive Reduction in Cholesterol Levels) trial was associated with a significantly increased risk of new-onset T2DM (8.71 vs $6.06 \%$, adjusted HR 1.37, 95\% CI: $1.08-1.75, \mathrm{p}=0.011)$. In the TNT (Treating to New Targets) trial (atorvastatin $80 \mathrm{mg}$ vs atorvastatin $10 \mathrm{mg}$ ) and in the IDEAL (Incremental Decrease in End Points Through Aggressive Lipid Lowering) study evaluating atorvastatin $80 \mathrm{mg}$ versus simvastatin 20 - $40 \mathrm{mg}$, the differences were trivial and not significant. Baseline fasting glucose levels and features of the metabolic syndrome were predictive of new-onset T2DM across the three trials [49]. Thus, even if atorvastatin (at high dosage) may somewhat deteriorate glucose control and increase the risk of new-onset diabetes, these effects appear relatively small and most probably not really clinically relevant, compared with the cardiovascular benefits provided by the drug.

If atorvastatin may slightly increase the risk of new-onset diabetes, it may also worsen glycemic control in patients with T2DM. In CARDS, a slightly greater rise in HbA1c was observed in the atorvastatin $10 \mathrm{mg}$ group (from 7.87 to $8.3 \%$ ) than in the placebo group (from 7.81 to 8.1\%) [9]. In ASPEN, a $0.2 \%$ increase in HbA1c was observed in both patients treated with atorvastatin $10 \mathrm{mg}$ and placebo after 4 years of follow-up [10]. More importantly, in the AFORRD study [50], a glycémie worsening potential of atorvastatin $20 \mathrm{mg}$ of about $0.3 \%$ HbA1c was noticed (+0.37\% vs $+0.09 \%$ in the placebo group; $\mathrm{p}<0.001)$. So in combination with the use of sitagliptin, which has a mean HbA1c-lowering capacity of 0.7 or $0.8 \%$ in randomized trials (see below), this might mean that part of the HbA1c-lowering effect of sitagliptin will be lost in combination with atorvastatin. This might be a concern, and a prospective randomized and placebo-controlled study looking into this issue is urgently needed.

Because of these findings in several studies, the FDA has added early 2012 wording to the 'warnings and precautions' section of statins: 'People being treated with statins may have an increased risk of raised blood sugar levels and the development of T2DM' [51].

Figure 1. Metabolic and cardiovascular effects of atorvastatin

CV: Cardiovascular; HMG-CoA: hydroxymethylglutaryl coenzyme A; LDL-C: LDL cholesterol; TG:

Triglycerides.

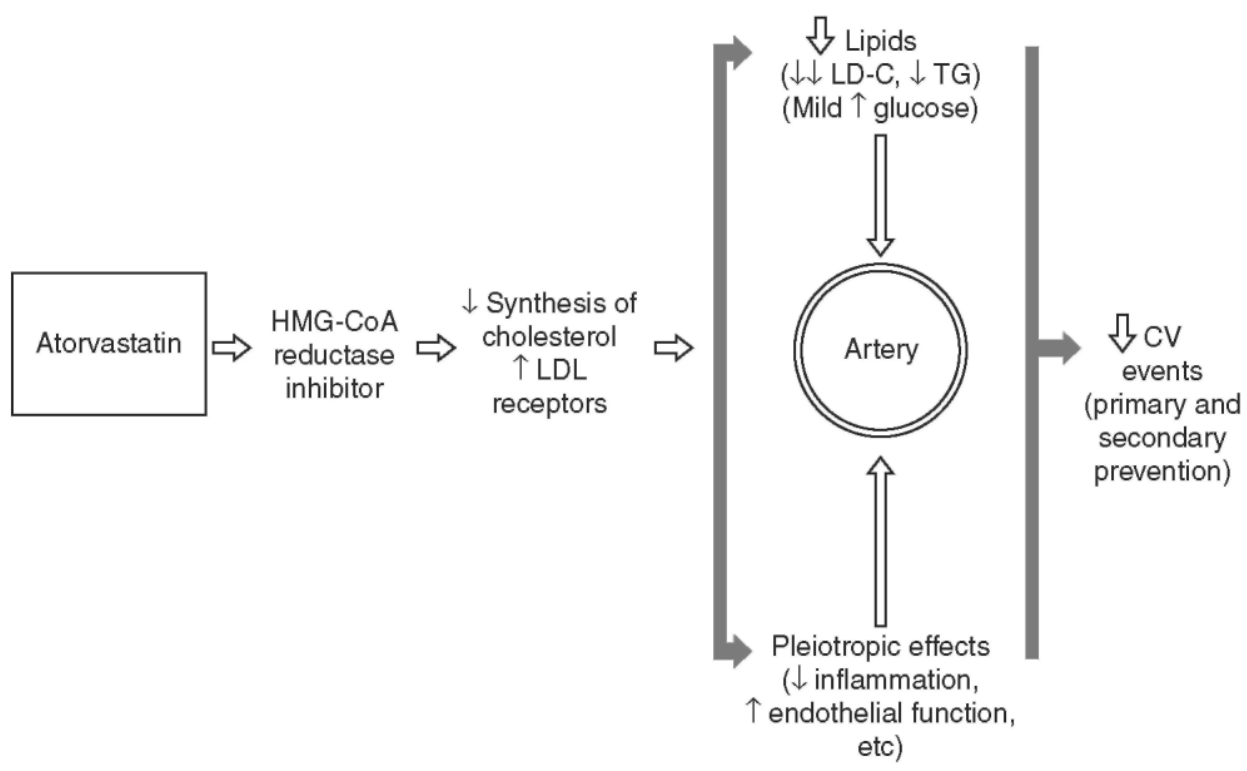

\subsection{Effect of sitagliptin on glucose and lipid metabolism}

\subsubsection{Sitagliptin and glucose control}

By selectively inhibiting DPP-4 enzyme activity, sitagliptin increases GLP-1 levels, which results in improving insulin secretion (incretin effect) and reducing glucagon secretion (Figure 2). Thereby, it reduces fasting (-1 to 2 $\mathrm{mmol} / \mathrm{l})$ and even more postprandial (-2 to $4 \mathrm{mmol} / \mathrm{l})$ plasma glucose levels. Sitagliptin has proven to be effective in patients with T2DM treated with diet alone, metformin, a sulfonylurea, a thiazolidinedione, a 
combination metformin-sulfonylurea or even insulin [22,23]. A synergistic effect has been particularly reported when added to metformin [24]. Overall, the reduction in HbA1c levels averages $0.8 \%(0.6-1.0 \%)$ when compared with placebo. Such a reduction is almost similar to that seen with other oral glucose-lowering agents, provided that basal HbA1c levels are taking into account for the comparison [52]. Of clinical interest, this improvement in blood glucose control with sitagliptin occurs without inducing hypoglycemia or weight gain $[22,23]$.

\subsubsection{Sitagliptin and lipid profile}

A recent meta-analysis suggested a possible beneficial effect of DPP-4 inhibitors on cholesterol, which, although small, could contribute to the reduction of CVD risk. Although the number of trials of appropriate size and duration was high $(n=53)$, only a small fraction of those $(n=17)$ reported data on endpoint total, HDL and LDL cholesterol and TG levels. The difference in means for endpoint versus baseline total cholesterol in patients on DPP-4 inhibitor treatment was significantly higher in comparison with controls, meaning that treatment with DPP-4 inhibitors is associated with a significant reduction in total cholesterol $(-0.18(-0.29 ;-0.06) \mathrm{mmol} / \mathrm{l}(-7.0$ $(-11.2 ;-2.50) \mathrm{mg} / \mathrm{dl}) ; \mathrm{p}=0.002)[53]$.

DPP-4 inhibitors have also been found to have an effect on postprandial lipid levels [54]. Treatment with sitagliptin for 6 weeks reduced postprandial plasma levels of TG-rich lipoproteins of both intestinal and hepatic origin, most likely by increasing incretin hormone levels, reducing circulating plasma free fatty acid concentrations and improving insulin sensitivity and $\beta$-cell function [55]. Overall, however, the effect of sitagliptin on lipid metabolism seems to be modest, although statistically significant: sitagliptin $100 \mathrm{mg}$ significantly decreased the postprandial AUCs for plasma apolipo-protein (apo)B (-5.1\%), TG (-9.4\%), VLDL cholesterol $(-9.3 \%)$ and free fatty acids $(-7.6 \%)$.

Figure 2. Metabolic and cardiovascular effects of sitagliptin.

CV: Cardiovascular; DPP-4: Dipeptidyl peptidase-4; GIP: Glucose-dependent insulinotropic polypeptide; GLP1: Glucagon-like peptide-1.

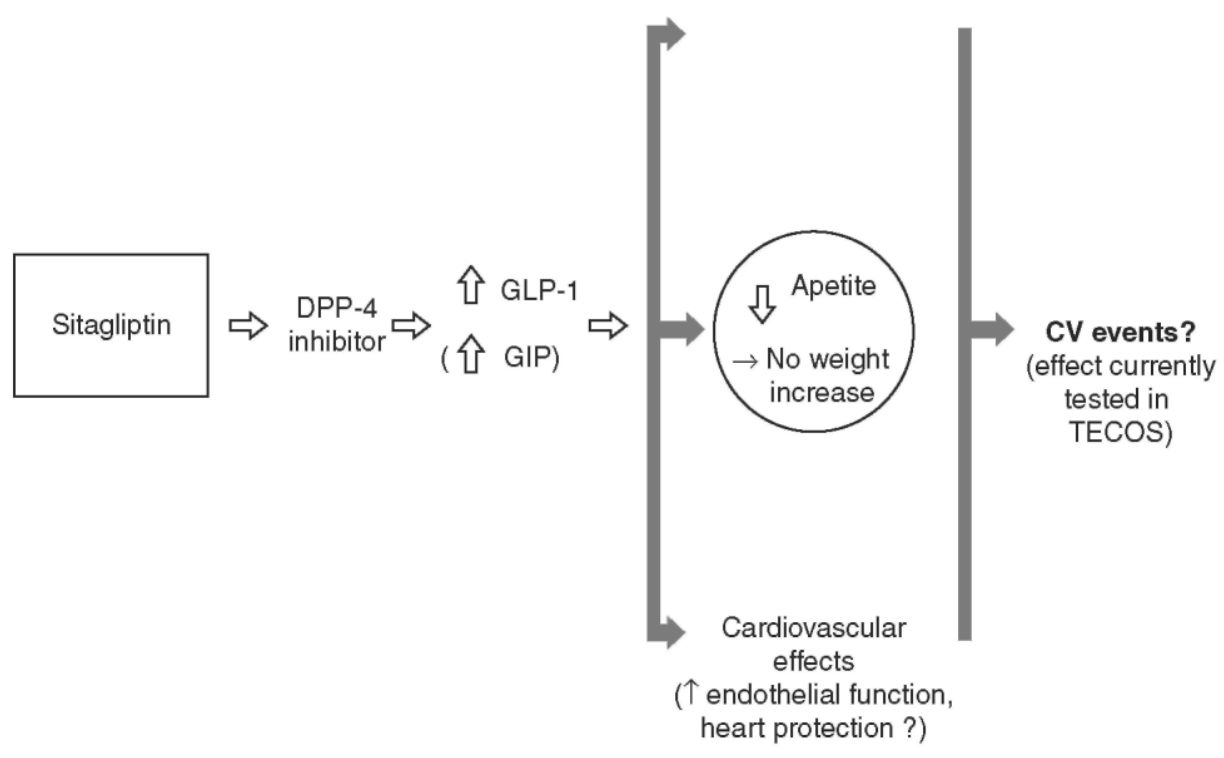

\section{Efficacy on cardiovascular disease}

Considering the complementary mechanisms of action of atorvastatin and sitagliptin, which result in tackling several cardiovascular risk factors, one may expect that combining the two pharmacological agents may improve CVD prognosis (Figure 3).

\subsection{Atorvastatin}

The introduction of statins has drastically changed the treatment and prevention of atherosclerotic vascular disease. By lowering lipid levels and exerting various pleiotropic effects (for instance, reduction in silent inflammation and improvement of endothelial function), these drugs are among the most effective at reducing 
CVD morbidity and mortality available to clinical practice [3]. A meta-analysis of data from 18,686 individuals with diabetes (1466 with type 1 and 17,220 with type 2) in 14 randomized trials of statin therapy demonstrated a significant $21 \%$ proportional reduction in major vascular events per mmol/l reduction in LDL cholesterol in people with diabetes $(0.79,95 \%$ CI $0.72-0.86)$, which was similar to the effect observed in those without diabetes [6]. These results were confirmed in a second metaanalysis from the same Cholesterol Treatment Trialists' Collaboration [3]. Across 26 trials on statin therapy, all-cause mortality was reduced by $10 \%$ per 1.0 $\mathrm{mmol} / \mathrm{L}$ LDL reduction (RR 0.90, 95\% CI $0.87-0.93 ; \mathrm{p}<0 \bullet 0001$ ), largely reflecting significant reductions in deaths due to coronary heart disease (RR $0.80,99 \%$ CI $0.74-0.87$ ) and other cardiac causes (RR 0.89, 99\% CI 0.81 - 0.98). Concerning the effect on major vascular events per $1.0 \mathrm{mmol} / \mathrm{l}$ reduction in LDL cholesterol, a RR of 0.80 (CI 95\% $0.74-0.86$ ) was found in patients with T2DM compared with 0.78 (CI 95\% $0.75-0.81$ ) in subjects without diabetes. There was no evidence of any threshold within the cholesterol range studied, suggesting that reduction of LDL cholesterol by $2-3 \mathrm{mmol} / \mathrm{l}$ would reduce risk by about $40-50 \%$.

However, the two meta-analyses of the Cholesterol Treatment Trialists consider primary prevention and secondary intervention together, which tends to obscure the evidence for true primary prevention, especially in patients with T2DM. A literature-based meta-analysis of 11 studies did not find evidence for the benefit of statin therapy on all-cause mortality (risk ratio, 0.91 ; 95\% CI 0.83 - 1.01) in a high-risk primary prevention set-up, including patients with T2DM [56]. A Cochrane review of 14 randomized control trials assessed the effects of statins in people with no history of CVD (primary prevention), with data collected in patients with or without diabetes [57]. All-cause mortality was reduced by statins (RR 0.83, 95\% CI 0.73 - 0.95) as was combined fatal and non-fatal CVD endpoints (RR 0.70, 95\% CI 0.61 - 0.79). However, the authors found some evidence of selective reporting of outcomes and inclusion of people with CVD. The conclusions were that only limited evidence showed that primary prevention with statins may be cost effective and that caution should be taken in prescribing statins for primary prevention among people at low cardiovascular risk [57].

Reductions in total and LDL cholesterol levels achieved with atorvastatin have been shown to translate into reductions in risk of cardiovascular morbidity and mortality in both primary and secondary prevention settings [58], although heterogeneous results were reported in the two trials that exclusively enrolled T2DM patients. In CARDS, atorvastatin $10 \mathrm{mg} /$ day was efficacious in reducing the risk of first CVD events (-37\%; $95 \%$ CI: -52 to -17; $\mathrm{p}=0.001)$ in patients with T2DM without high LDL cholesterol. Assessed separately, acute coronary heart disease events were reduced by $36 \%$ ( -55 to -9$)$, coronary revascularizations by $31 \%$ (-59 to 16) and rate of stroke by $48 \%$ ( -69 to -11 ). Atorvastatin reduced the death rate by $27 \%$ ( -48 to $1, p=0.059$ ) [9]. In ASPEN, despite a mean LDL cholesterol reduction of $29 \%$ versus placebo in the atorvastatin $10 \mathrm{mg}$ group over 4 years, the composite primary endpoint incidence was not significantly reduced in the whole cohort (hazard ratio 0.90 95\% CI 0.73 - 1.12), neither in T2DM patients without prior myocardial infarction or interventional procedure (primary prevention : hazard ratio $0.97 ; 0.74-1.28$ ), nor in patients with prior myocardial infarction or interventional procedure (secondary prevention : hazard ratio $0.82 ; 0.59-1.15$ ) [10]. The reasons for different effects in ASPEN when compared with CARDS are not clear, but may relate to the overall study design, the types of subjects recruited, the nature of the primary endpoint and the protocol changes required because of changing treatment guidelines. The conclusion was that the results of ASPEN did not confirm the benefit of therapy with atorvastatin but do not detract from the imperative that the majority of diabetic patients are at risk of coronary heart disease and deserve LDL cholesterol lowering to the currently recommended targets [10]. In this regard, atorvastatin has a potential role in the primary prevention of CVD events in diabetic patients at risk, irrespective of pretreatment LDL-cholesterol levels [59]. Nevertheless, the debate is still open in primary prevention as pointed out in a recent systematic review (including CARDS and ASPEN results, besides other trials) whose results questioned the use of cholesterol lowering to reduce mortality and cardiovascular complications in T2DM [60].

\subsection{Sitagliptin}

T2DM is a well-established risk factor for CVD [1,2], and new therapeutic approaches, such as incretin-based therapies, should ideally also target CVD risk, beyond glucose control [61]. In a meta-analysis of 41 RCTs (9 of which are unpublished), the risk of CVD events and all-cause death with DPP-4 inhibitors was 0.76 (95\% CI $0.46-1.28)$ and $0.78(0.40-1.51)$, respectively [62]. A pooled analysis more specifically included data from 19 double-blind, randomized studies including 10,246 T2DM patients who received either sitagliptin $100 \mathrm{mg} / \mathrm{day}$ or a comparator agent (placebo or an active comparator). Treatment with sitagliptin was not associated with an increased risk of major adverse cardiovascular events versus comparator but rather with a lower incidence of CVD complications (0.6 vs $0.9 \%$ ). In the prespecified analysis, the risk ratio for sitagliptin-exposed relative to non-exposed patients was 0.68 (95\% CI: 0.41, 1.12) [63]. Recent experimental data suggested that the DPP-4 inhibitor sitagliptin may exert cardiovascular protective effects beyond its effects on glucose metabolism [64]. 
Figure 3. Complementary metabolic and vascular mechanisms of action of atorvastatin and sitagliptin, which may result in improving cardiovascular prognosis in patients with type 2 diabetes.

CV: Cardiovascular

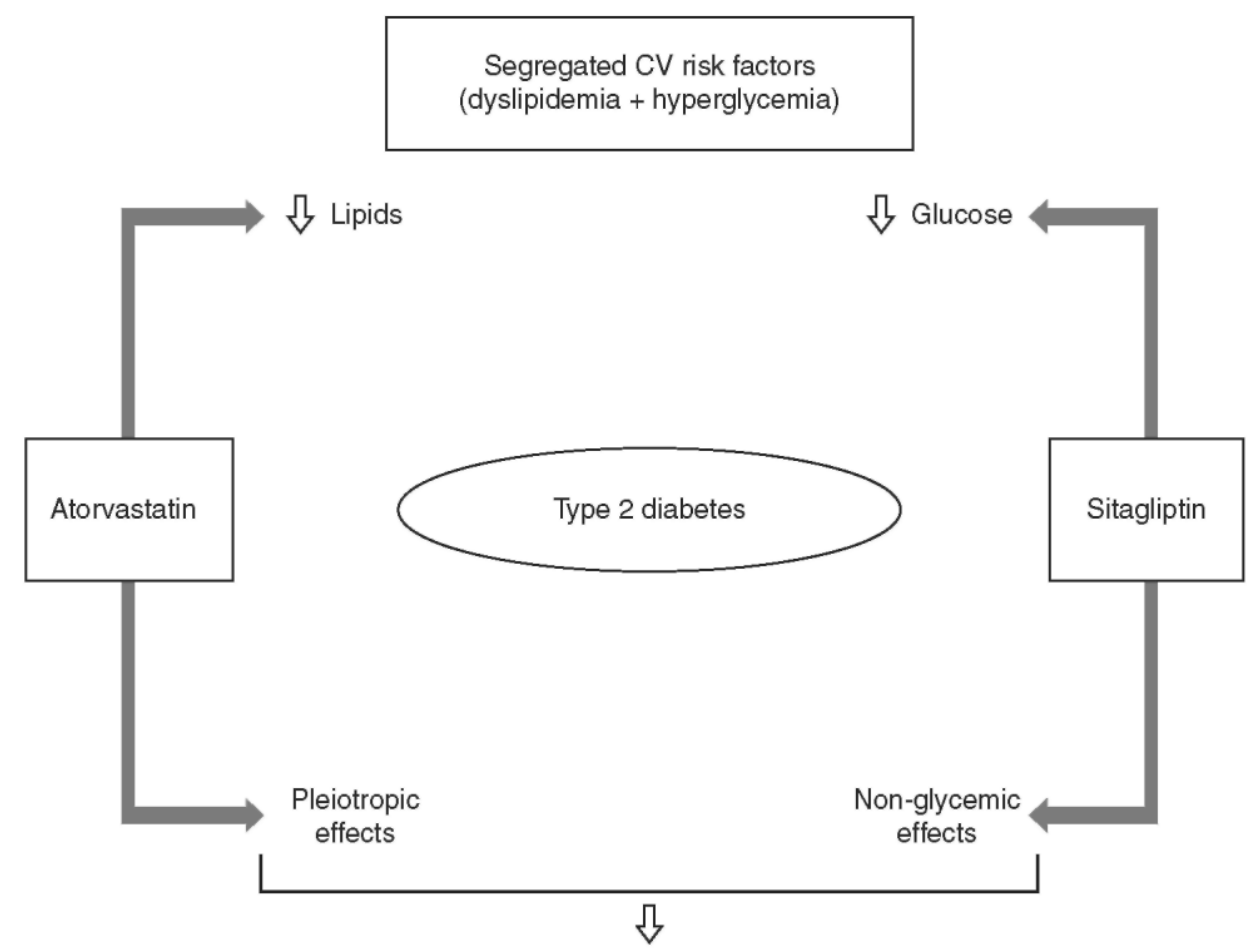

Whether gliptins actually decrease CVD outcomes remains to be confirmed by large randomized placebocontrolled trials. Several prospective trials are ongoing in order to demonstrate the cardiovascular safety (most trials have, as first objective, to show non-inferiority cardiovascular outcome vs placebo) and possibly the superiority of DPP-4 inhibitors to reduce the incidence of CV events in T2DM patients at high risk of CVD. TECOS (Trial Evaluating Cardiovascular Outcomes with Sitagliptin: NCT00790205) is a long-term, eventdriven, non-inferiority cardiovascular outcomes trial specifically evaluating the impact of sitagliptin when added to usual diabetes care in a large, high-risk population [65]. Its primary objective is to determine whether including sitagliptin as part of usual care has any impact on a composite cardiovascular endpoint (cardiovascular-related death, nonfatal heart attack, nonfatal stroke, or unstable chest pain or discomfort of cardiac origin requiring hospitalization). This multinational, placebo-controlled, double-blind, randomized, parallel-group clinical trial will enroll approximately 14,000 patients, with an approximate follow-up of four to five years until 1300 primary cardiovascular events are accumulated. Study participants will have T2DM and preexisting CVD, be at least 50 years of age and have inadequate glycemic control (baseline HbA1c $=6.5-$ $8.0 \%$ ) despite monotherapy or dual combination therapy with metformin, pioglitazone or a sulfonylurea. Full glycemic and cardiovascular risk factor treatment according to local guidelines for all participants is being emphasized. Because of the profile of the patients enrolled in TECOS, almost all of them will be treated by statin and, because of the common use of atorvastatin in clinical practice, presumably numerous will receive atorvastatin at various doses. Thus, this large trial will represent a great opportunity to test in the long run both the efficacy and safety of the atorvastatin and sitagliptin association in a quite huge number of T2DM patients at high CVD risk.

\subsection{Atorvastatin-sitagliptin coadministration}

Reduction of CVD events in patients with T2DM requires a global approach targeting all risk factors [66]. As many patients, especially with T2DM, present both arterial hypertension and dyslipidemia, atorvastatin has been associated with the dihydropyridine calcium channel antagonist amlodipine. Amlodipine/atorvastatin as a singletablet, FDC (Caduet) offers a convenient and effective approach to improving adherence and managing risk in hypertensive patients with dyslipidemia or at risk of CVD [30]. 
Because of the well-known positive effects of atorvastatin and the promising results already reported with sitagliptin, we can speculate that the dual therapy should exert a favorable action on metabolic profile and CVD outcomes (Figure 3). However, this is not proven yet and to our knowledge, there is no ongoing trial specifically testing this hypothesis. Currently, only short-term clinical trials are testing the efficacy on metabolic endpoints and overall safety of combining atorvastatin and sitagliptin in patients with T2DM. A Phase III study (NCT01477853) has begun enrolment of 750 patients with inadequately controlled T2DM on metformin monotherapy.

The study will examine if a treatment with sitagliptin in combination with atorvastatin reduces HbA1c and LDL cholesterol from baseline more than atorvastatin alone and sitagliptin alone, respectively. Following a singleblind, placebo run-in period, participants will be randomized to one of three treatment arms (sitagliptin monotherapy with placebo to atorvastatin, atorvastatin monotherapy with placebo to sitagliptin or sitagliptin plus atorvastatin) for 16 weeks (Phase A). During Phase B of the study (weeks 16 through 54), participants will receive either sitagliptin plus atorvastatin with placebo to glimepiride or glimepiride plus atorvastatin with placebo to sitagliptin. The study is expected to complete in 2014.

\section{Safety profile}

\subsection{Atorvastatin}

The existing data suggest that atorvastatin is generally well tolerated across the range of its therapeutic dosage $(10-80 \mathrm{mg} /$ day) [67]. Withdrawal rates related to adverse events are low $(<$ or $=3 \%)$. The most common adverse events are mild gastrointestinal symptoms. Elevated serum transaminase levels occur infrequently $(<$ or $=1.5 \%)$. These are generally asymptomatic, reversible and rarely require drug withdrawal. However, regular monitoring of liver enzymes is still recommended in most recent guidelines [5]. Dose-related myopathy and/or rhabdomyolysis also occurs very rarely, although the risk is increased by concomitant administration of cyclosporine, niacin, fibrates or by CYP3A4 isoenzyme inhibitors (e.g., erythromycin, systemic azole antifungal agents, etc.) with statins metabolized by this isoenzyme [21]. The PK of the individual statin, in the present case atorvastatin, should be considered in patients receiving polypharmacological treatments, to minimize the risk of unfavorable drug interactions [68]. Nevertheless, as already discussed, because of individual PK characteristics of the two compounds, the risk of DDIs between atorvastatin and sitagliptin appears almost negligible.

\subsection{Sitagliptin}

The safety profile of sitagliptin is in general excellent $[22,23]$. In a recently updated pooled safety analysis of data from 10,246 patients with T2DM, sitagliptin $100 \mathrm{mg}$ /day was generally well tolerated in clinical trials of up to 2 years in duration [63]. In particular, no notable differences in incidence rates were observed for anyinfection-related adverse events between the group of patients treated with sitagliptin and the group of patients not exposed to sitagliptin. The increased incidence rate of skin-related adverse events overall observed in the sitagliptin group was due to small differences in a few adverse events, including contact dermatitis and rash. However, a higher rate of angioedema or angioedema-related events was not observed in sitagliptin-treated patients compared with patients not exposed to sitagliptin. Nevertheless, a higher risk might be suspected when the DPP-4 inhibitor is coadministered with an angiotensin-converting enzyme inhibitor (a pharmacological class also frequently used in T2DM patients at high CVD risk) because both inhibitors are able to block the degradation of bradykinin and substance $\mathrm{P}$, that is, two proinflammatory peptides [69]. In this pooled analysis, in which $82 \%$ of patients had cardiovascular risk factors in addition to diabetes and CVD, there was no difference between groups in the evaluation of cardiovascular complications overall, but a trend for a lower incidence of major ischemic events in the sitagliptin-treated group than in the non-exposed group, as already mentioned [63]. Finally, the same pooled analysis of controlled clinical trials revealed similar incidence rates of pancreatitis in patients treated with sitagliptin compared with those not treated with sitagliptin $(0.08$ events per 100 patientyears vs 0.10 events per 100 patient-years, respectively) [70]. Nevertheless, post-marketing careful check of the incidence of pancreatitis with incretin-based therapies, including sitagliptin, is still recommended.

\subsection{Atorvastatin-sitagliptin coadministration}

There is no obvious reason to consider that the dual therapy atorvastatin plus sitagliptin may be harmful. In clinical practice, the two drugs are commonly prescribed in patients with T2DM without any reported adverse events. Especially, liver enzymes do no appear to be altered by the combination atorvastatin and sitagliptin. However, an increased risk for muscle toxicity has been suspected after the report of a few case reports with atorvastatin and other statins. 
A case of rhabdomyolysis in a 75-year-old Caucasian man with T2DM taking atorvastatin for several years who had recently (6 months ago) started taking sitagliptin. Unfortunately the doses of the two compounds were not mentioned in the letter to the editor [71]. Rhabdomyolysis was defined here as the breakdown of muscle tissue (evidenced by muscle pain and huge increase in serum creatinine kinase levels) attributable to any cause (no other cause outside drug therapy could be identified in this clinical case), which can but does not necessarily predispose to acute kidney injury (which was not the case here as renal function was and remained within normal range). Magnetic resonance imaging of the thighs showed perimuscular edema consistent with an inflammatory myopathy (muscle biopsy was not performed). Both sitagliptin and atorvastatin were stopped promptly and intravenous crystalloid was administered, and the clinical and biological evolution was progressively favorable. The authors concluded that sitagliptin and atorvastatin should be coadministered with caution. Of note, two other cases of rhabdomyolysis have been reported with the combination of sitagliptin and other HMG-CoA reductase inhibitors, simvastatin [72] and lovastatin [73], respectively. The case involving simvastatin concerned a 76year-old man with chronic kidney disease receiving numerous medications, including amiodarone, olmesartan and diuretics. The authors concluded that high doses of sitagliptin (100 mg per day) in the presence of chronic kidney disease may have worsened this patient's renal failure and precipitated rhabdomyolysis by increasing circulating levels of simvastatin [72]. However, the causal relationship of sitagliptin added to simvastatin in the muscular toxicity has been debated, especially because of concomitant therapy with amiodarone in this patient [74]. In the other case, a possible interaction between sitagliptin and lovastatin at the level of the cytochrome P450 enzyme system was suspected. Indeed, both drugs are metabolized by the enzyme CYP3A4 [32,35], and when coadministered, sitagliptin could theoretically increase the plasma concentration of lovastatin by competition for the enzyme. This would increase the likelihood of lovastatin causing muscle toxicity. A similar mechanism could not be excluded with a coadministration of atorvastatin and sitagliptin. Nevertheless, these cases are rather exceptional so that one may question the need for a close monitoring for clinical and biochemical evidence of toxic myopathy in patients taking statins (atorvastatin) and DPP-4 inhibitor sitagliptin). Check of serum creatine kinase levels is only recommended in case of clinical symptoms evoking muscular toxicity [5].

Because of the PK characteristics of sitagliptin [37], to achieve plasma concentrations similar to those observed in patients with normal renal function treated with $100 \mathrm{mg}$ sitagliptin once daily, patients with moderate RI should receive sitagliptin $50 \mathrm{mg}$ once daily and patients with severe RI $25 \mathrm{mg}$ once daily. When using such dose adjustment, sitagliptin was generally well tolerated and provided effective glycemic control in patients with T2DM and moderate to severe RI [75]. Furthermore, a recent retrospective cohort study of a large medical and pharmacy claims database revealed an increased incidence of acute renal failure in diabetic versus non-diabetic patients but no association between use of sitagliptin and acute renal failure [76].

\section{Conclusion}

Patients with T2DM are exposed to a considerably high CVD risk, which requires a global management to improve prognosis. Atorvastatin, a potent lipid-lowering agent, and sitagliptin, a glucose-lowering agent belonging to the incretin-based pharmacological class, can be used together in order to provide a global cardiovascular protection in patients with T2DM. Considering the individual PK characteristics, no adverse DDIs may be expected from the combination of the two drugs. No proven special adverse events have been reported with the association of atorvastatin and sitagliptin yet. By reducing LDL cholesterol and glucose levels, several favorable vascular effects may be expected, for instance, reduction in silent inflammation and improvement of endothelial function. Further studies should demonstrate the clinical value of such a combination. In this regard, the results of the ongoing large prospective cardiovascular outcomes trial TECOS will probably be of great interest. An FDC may be considered although its place in clinical practice remains to be demonstrated, especially when considering the already available classical sitagliptin-metformin combination and amlodipine-atorvastatin combination in the US market.

\section{Expert opinion}

The cardiovascular complications of T2DM represent a major burden for patients and health care systems. Therefore, everybody will agree that prevention of CVD is a major objective in the management of patients with T2DM. To reach this objective, a global management targeting not only hyperglycemia but also all other risk factors, including dyslipidemias, is strongly recommended. In the recommendations of the American Diabetes Association, statin therapy should be added to lifestyle therapy, regardless of baseline lipid levels, for diabetic patients with overt CVD (Evidence level A) and without CVD who are over the age of 40 years and have one or more other CVD risk factors (Evidence level A). For lower-risk patients than the above (e.g., without overt CVD and under the age of 40 years), statin therapy should also be considered in addition to lifestyle therapy if LDL cholesterol remains $>2.5 \mathrm{mmol} / \mathrm{l}(100 \mathrm{mg} / \mathrm{dl})$ or in those with multiple CVD risk factors (Evidence level E). In 
the recent guidelines from the European Society of Cardiology and the European Atherosclerosis Society, all patients with T2DM should receive statin therapy, except perhaps patients younger than 40 years, with a short duration of therapy, without other risk factors, without complications and with an LDL cholesterol $<2.5 \mathrm{mmol} / 1$ $(100 \mathrm{mg} / \mathrm{dl})$ who may not need lipid-lowering drugs (Evidence level B). Thus, a large majority of T2DM patients should receive a statin so that such pharmacological intervention obviously is in the heart of T2DM global management. Interestingly enough, in CARDS, atorvastatin, at a low dose of $10 \mathrm{mg}$ per day, has proven its efficacy and safety in patients with T2DM, with a 37\% reduction in first CVD events compared with placebo despite the absence of high cholesterol concentrations. Because of these favorable results, CARDS was discontinued 2 years before the anticipated end. In the absence of significant effect on both overall and cardiovascular mortality, one may question whether the trial should not have been prematurely stopped, especially in view of the nonsignificant reduction of cardiovascular events in ASPEN, another study also having evaluated the effects of atorvastatin $10 \mathrm{mg}$ in patients with T2DM.

Controlling hyperglycemia without inducing hypoglycemia (and weight gain) remains a big challenge in the field of diabetes management. In this regard, incretin-based therapies raised considerable interest in recent years. Besides GLP-1 mimetics or analogs, DPP-4 inhibitors are a promising pharmacological class of glucoselowering agents that open new perspectives for the management of T2DM. By inhibiting GLP-1 degradation and increasing circulating levels, thereby promoting insulin secretion and reducing glucagon secretion, both in a glucose-dependent manner, these agents are characterized by a mechanism of action that is distinct from (and also complementary to) any existing class of oral glucose-lowering agents. Because of their unique mechanism of action, these new oral agents meet the, until recently, unmet needs of improving glucose control without hypoglycemia and weight gain. Furthermore, positive effects beyond glucose control have been reported, both in animal models and in humans, which may contribute to improve endothelial dysfunction and exert cardiac protective effects. Preliminary retrospective results from Phase III studies suggested a possible reduction in CVD events in patients receiving DPP-4 inhibitors, including sitagliptin. These encouraging observations trigger ongoing large prospective trials, which should first confirm the safety of DPP-4 inhibitors and second, hopefully, show some cardiovascular benefits in addition to underlying basal therapy. In this regard, sitagliptin will be evaluated in TECOS versus placebo, added on top of other glucose-lowering agents (except other incretin therapies) and lipid-lowering medications. However, because of the design of the study, which allows any clinically relevant pharmacological adjustment during the rather long-term follow-up (almost 4-5 years), it is to be feared that the demonstration of a significant reduction of CVD events with sitagliptin in such a trial will be difficult to be achieved (therefore, the non-inferiority design of the study).

By tackling both dyslipidemia (with atorvastatin) and hyperglycemia (with sitagliptin), without worsening other risk factors, one may expect an additive or even a synergistic effect in the prevention of CVD events in patients with T2DM. This is especially true because both compounds have also shown so-called pleiotropic effects, potentially favorable to both vessels and heart, beyond their lipid and glucose effects, respectively. One recent concern, however, was the demonstration that statin therapy may negatively interfere with glucose metabolism and increase the risk of new-onset diabetes. Controversial findings have been reported, with a reduced risk for pravastatin contrasting with an increased risk for other statins, including atorvastatin. Nevertheless, if present, this negative metabolic effect appears rather small (considering the high number needed to harm, around 250), and this effect should be weighed against the favorable protective role of statins against CVD, the number one killer of patients with T2DM.

A final comment may be devoted to the place of such an atorvastatin-sitagliptin association if an FDC would be available on the market in the future. At first glance, such a combination may appear appealing from a PD but also PK point of view. Indeed, the two pharmacological agents are given once daily and do not show deleterious DDIs. However, some concerns should be raised. First of all, it should be pointed out that until now there is no available study (at least published as full papers referred in PUBMED) having specifically evaluated the combination of atorvastatin and sitagliptin, either separately (which is quite astonishing) or of course as an FDC. Furthermore, two different FDCs are already available, the atorvastatin-amlodipine FDC (at least in the US) and the sitagliptin-metformin FDC (worldwide). Because metformin is considered as the first-line therapy in the management of T2DM, an atorvastatin-sitagliptin FDC, if available for clinicians, would be in competition with the sitagliptin-metformin FDC. Thus, we can speculate that a new atorvastatin-sitagliptin FDC might preferably be used in T2DM patients in whom metformin is either contraindicated (RI) or not tolerated (gastrointestinal adverse events). In case of RI, the dose of sitagliptin should be reduced according to glomerular filtration rate because this DPP-4 inhibitor is mainly excreted via the kidney. This is not the case for atorvastatin, which is essentially eliminated by the liver. Thus, the prescription of an atorvastatin-sitagliptin FDC in patients with RI should take into account this dose adjustment of the DPP-4 inhibitor, which may limit the easy use of such an FDC in patients with T2DM at risk of RI, as in elderly patients. Finally, regarding an FDC of 
atorvastatin/sitagliptin, also the application modalities need to be discussed in relation to meal intake and daytime intake, which might be crucial for efficacy. Again this might need evaluation in prospective controlled and randomized trials. Thus, further studies are awaited to validate the use and to better describe the place of such an atorvastatin-sitagliptin combination in clinical practice.

\section{Declaration of interest}

AJ Scheen declares that he has no conflicts of interest and has received no payment in the preparation of this manuscript. He, however, declares that he has received lecture/advisor fees from AstraZeneca, Bristol-Myers Squibb, Boehringer Ingelheim, Eli Lilly, GlaxoSmithKline, Merck Sharp \& Dohme, Novartis, Novo Nordisk, Sanofi-Aventis and Sender.

\section{Bibliography}

Papers of special note have been highlighted as either of interest $(\cdot)$ or of considerable interest $(\cdot \cdot)$ to readers.

1. Ryden L, Standi E, Bartnik M, et al. Guidelines on diabetes, pre-diabetes, and cardiovascular diseases: executive summary. The Task Force on Diabetes and cardiovascular diseases of the European Society of Cardiology (ESC) and of the European Association for the Study of Diabetes (EASD). Eur Heart J 2007;28:88-136

2. Buse JB, Ginsberg HN, Bakris GL, et al. Primary prevention of cardiovascular diseases in people with diabetes mellitus: a scientific statement from the American Heart Association and the American Diabetes Association. Diabetes Care 2007;30:162-72

3. Baigent C, Blackwell L, Emberson J. et al. Efficacy and safety of more intensive lowering of LDL cholesterol: a meta-analysis of data from 170,000 participants in 26 randomised trials. Lancet 2010;376:1670-81

4. Association AD. Standards of medical care in diabetes - 2012. Diabetes Care 2012;35(Suppl 1):S11-63

5. Reiner Z, Catapano AL, De Backer G. et al. ESC/EAS Guidelines for the management of dyslipidaemias: the Task Force for the management of dyslipidaemias of the European Society of Cardiology (ESC) and the European Atherosclerosis Society (EAS). Eur Heart J $2011 ; 32: 1769-818$

6. Kearney PM, Blackwell L, Collins R et al. Efficacy of cholesterol-lowering therapy in 18,686 people with diabetes in 14 randomised trials of statins: a meta-analysis. Lancet 2008;371:117-25

7. Lea AP, McTavish D. Atorvastatin. A review of its pharmacology and therapeutic potential in the management of hyperlipidaemias Drugs 1997;53:828-47

8. Malhotra HS, Goa KL. Atorvastatin: an updated review of its pharmacological properties and use in dyslipidaemia. Drugs $2001 ; 61: 1835-8$

9. Colhoun HM, Betteridge DJ, Durrington PN, et al. Primary prevention of cardiovascular disease with atorvastatin in type 2 diabetes in the Collaborative Atorvastatin Diabetes Study (CARDS): multicentre randomised placebo-controlled trial. Lancet 2004;364:685-96

This study demonstrated that atorvastatin $10 \mathrm{mg} / \mathrm{day}$ is safe and efficacious in reducing the risk of first CVD events, including stroke, in patients with T2DM without high LDL cholesterol.

10. Knopp RH, d'Emden M, Smilde JG, et al. Efficacy and safety of atorvastatin in the prevention of cardiovascular end points in subjects with type 2 diabetes: the Atorvastatin Study for Prevention of Coronary Heart Disease Endpoints in non-insulin-dependent diabetes mellitus (ASPEN). Diabetes Care 2006;29:1478-85

11. Sarwar N, Gao P, Seshasai SR, et al. Diabetes mellitus, fasting blood glucose concentration, and risk of vascular disease: a collaborative meta-analysis of 102 prospective studies. Lancet 2010;375:2215-22

12. Ceriello A, Hanefeld M, Leiter L, et al. Postprandial glucose regulation and diabetic complications. Arch Intern Med 2004;164:2090-

13. Yakubovich N, Gerstein HC. Serious cardiovascular outcomes in diabetes: the role of hypoglycemia. Circulation 2011;123:342-8

14. Bonds DE, Miller ME, Bergenstal RM, et al. The association between symptomatic, severe hypoglycaemia and mortality in type 2 diabetes: retrospective epidemiological analysis of the ACCORD study. BMJ 2010;340:b4909

15. Seaquist ER, Miller ME, Bonds DE. et al. The impact of frequent and unrecognized hypoglycemia on mortality in the ACCORD study. Diabetes Care 2012;35:409-14

16. Scheen AJ. A review of gliptins in 2011. Expert Opin Pharmacother 2012;13:81-99

17. Gallwitz B, Haring HU. Future perspectives for insulinotropic agents in the treatment of type 2 diabetes-DPP-4 inhibitors and sulphonylureas. Diabetes Obes Metab 2010;12:1-11

18. Burge MR, Sood V, Sobhy TA, et al. Sulphonylurea-induced hypoglycaemia in type 2 diabetes mellitus: a review. Diabetes Obes Metab 1999;1:199-206

19. Scheen AJ. Drug interactions of clinical importance with antihyperglycaemic agents - an update. Drug Saf 2005;28:601-31

20. Scheen AJ. Dipeptidylpeptidase-4 inhibitors (gliptins): focus on drug-drug interactions. Clin Pharmacokinet 2010;49:573-88

21. Scheen AJ. Cytochrome P450-mediatec! cardiovascular drug interactions. Expert Opin Drug Metab Toxicol 2011;7:1065-82 
Published in: Expert Opinion on Drug Metabolism \& Toxicology (2012), vol. 8, iss. 6, pp. 745-758.

Status: Postprint (Author's version)

22. Lyseng-Williamson KA. Sitagliptin. Drugs 2007;67:587-97

23. Dhillon S. Sitagliptin: a review of its use in the management of type 2 diabetes mellitus. Drugs 2010;70:489-512

24. Scheen AJ. Pharmacokinetic and pharmacodynamic evaluation of sitagliptin plus metformin. Expert Opin Drug Metab Toxicol 2010;6:1265-76

25. Chwieduk CM. Sitagliptin/metformin fixed-dose combination: in patients with type 2 diabetes mellitus. Drugs 2011;71:349-61

26. Hutchins V, Zhang B, Fleurence RL. et al. A systematic review of adherence, treatment satisfaction and costs, in fixed-dose combination regimens in type 2 diabetes. Curr Med Res Opin 2011;27:1157-68

27. Rozenfeld Y, Hunt JS, Plauschinat C. et al. Oral antidiabetic medication adherence and glycemic control in managed care. Am J Manag Care 2008;14:71-5

28. Cramer JA. A systematic review of adherence with medications for diabetes. Diabetes Care 2004;27:1218-24

29. Bailey CJ, Day C. Fixed-dose single tablet antidiabetic combinations. Diabetes Obes Metab 2009;11:527-33

30. Curran MP. Amlodipine/Atorvastatin: a review of its use in the treatment of hypertension and dyslipidaemia and the prevention of cardiovascular disease. Drugs 2010;70:191-213

- This review paper showed that amlodipine/atorvastatin, as a fixed-dose combination (FDC), offers a convenient and effective approach to improving adherence and managing cardiovascular risk in hypertensive patients with dyslipidemia or at risk of CVD, a finding that possibly paves the route for another FDC (atorvastatin-sitagliptin).

31. Lennernas H. Clinical pharmacokinetics of atorvastatin. Clin Pharmacokinet 2003;42:1141-60

32. Neuvonen PJ, Niemi M, Backman JT. Drug interactions with lipid-lowering drugs: mechanisms and clinical relevance. Clin Pharmacol Ther 2006;80:565-81

33. Scheen AJ. Pharmacokinetics of dipeptidylpeptidase-4 inhibitors. Diabetes Obes Metab 2010;12:648-58

34. Bergman A, Ebel D, Liu F, et al. Absolute bioavailability of sitagliptin. an oral dipeptidyl peptidase-4 inhibitor, in healthy volunteers. Biopharm Drug Dispos 2007;28:315-22

35. Vincent SH, Reed JR, Bergman AJ, et al. Metabolism and excretion of the dipeptidyl peptidase 4 inhibitor [14C] sitagliptin in humans. Drug Metab Dispos 2007;35:533-8

36. Chu XY, Bleasby K, Yabut J, et al. Transport of the dipeptidyl peptidase-4 inhibitor sitagliptin by human organic anion transporter 3, organic anion transporting polypeptide 4C1, and multidrug resistance P-glycoprotein. J Pharmacol Exp Ther 2007;321:673-83

37. Bergman AJ, Cote J, Yi B, et al. Effect of renal insufficiency on the pharmacokinetics of sitagliptin, a dipeptidyl peptidase-4 inhibitor. Diabetes Care 2007;30:1862-4

38. Chung M, Calcagni A, Glue P, et al. Bioavailability of amlodipine besylate/ atorvastatin calcium combination tablet. J Clin Pharmacol 2006;46:1030-7

39. Bergman AJ, Cote J, Maes A, et al. Effect of sitagliptin on the pharmacokinetics of simvastatin. J Clin Pharmacol 2009;49:483-8

- This pharmacokinetic study demonstrated that there is no drug-drug interaction between sitagliptin and simvastatin, another HMG-CoA reductase inhibitor that may serve as reference for atorvastatin.

40. KP A, Meda VS, Raj Kucherlapati VSP, et al. Pharmacokinetic drug interaction between gemfibrozil and sitagliptin in healthy Indian male volunteers. Eur J Clin Pharmacol 2012;68:709-14

41. Wolffenbuttel BH, Franken AA Vincent HH. Cholesterol-lowering effects of rosuvastatin compared with atorvastatin in patients with type 2 diabetes - CORALL study. J Intern Med 2005;257:531-9

42. Group DALIDS. The effect of aggressive versus standard lipid lowering by atorvastatin on diabetic dyslipidemia: the DALI study: a double-blind, randomized. placebo-controlled trial in patients with type 2 diabetes and diabetic dyslipidemia. Diabetes Care 2001;24:1335-41

43. Sattar N, Preiss D, Murray HM, et al. Statins and risk of incident diabetes: a collaborative meta-analysis of randomised statin trials Lancet 2010;375:735-42

44. Koh KK, Sakuma I, Quon MJ. Differential metabolic effects of distinct statins. Atherosclerosis 2011;215:1-8

45. Kostapanos MS, Liamis GL. Milionis HJ, et al. Do statins beneficially or adversely affect glucose homeostasis? Curr Vase Pharmacol 2010;8:612-31

46. Baker WL, Talati R, White CM, et al. Differing effect of statins on insulin sensitivity in non-diabetics: a systematic review and metaanalysis. Diabetes Res Clin Pract 2010;87:98-107

47. Koh KK, Quon MJ, Han SH, et al. Atorvastatin causes insulin resistance and increases ambient glycemia in hypercholesterolemic patients. J Am Coll Cardiol 2010;55:1209-16

48. Simsek S, Schalkwijk CG. Wolffenbuttel BH. Effects of rosuvastatin and atorvastatin on glycaemic control in Type 2 diabetes-the CORALL study. Diabet Med 2012;29:628-31

49. Waters DD, Ho JE, DeMicco DA, et al. Predictors of new-onset diabetes in patients treated with atorvastatin: results from 3 large randomized clinical trials. J Am Coll Cardiol 2011;57:1535-45

50. Holman RR, Paul S, Farmer A, et al. Atorvastatin in Factorial with Omega-3 EE90 Risk Reduction in Diabetes (AFORRD): a randomised controlled trial. Diabetologia 2009;52:50-9

51. Drug Safety Communication: Important safety label changes to cholesterol-lowering statin drugs. FDA U.S. Drug and Food 
Administration. 2012.Available from: http://www.fda.gov/ Drugs/DrugSafety/ucm293101.htm [Last accessed 16 April 2012]

52. Scheen AJ. DPP-4 inhibitors in the management of type 2 diabetes : a critical review of head-to-head trials. Diabetes Metab 2012;38:89-101

53. Monami M, Lamanna C, Desideri CM. et al. DPP-4 inhibitors and lipids: systematic review and meta-analysis. Adv Ther 2012;29:14-

54. Baetta R, Corsini A. Pharmacology of dipeptidyl peptidase-4 inhibitors: similarities and differences. Drugs 2011;71:1441-67

55. Tremblay AJ, Lamarche B, Deacon CF. et al. Effect of sitagliptin therapy on postprandial lipoprotein levels in patients with type 2 diabetes. Diabetes Obes Metab 2011;13:366-73

56. Ray KK, Seshasai SR, Erqou S, et al. Statins and all-cause mortality in high-risk primary prevention: a meta-analysis of 11 randomized controlled trials involving 65,229 participants. Arch Intern Med 2010;170:1024-31

57. Taylor F, Ward K, Moore TH, et al. Statins for the primary prevention of cardiovascular disease. Cochrane Database Syst Rev 2011;1:CD004816

58. van Leuven SI, Kastelein JJ. Atorvastatin. Expert Opin Pharmacother 2005;6:1191-203

59. Croom KF, Plosker GL. Atorvastatin: a review of its use in the primary prevention of cardiovascular events in patients with type 2 diabetes mellitus. Drugs 2005;65:137-52

60. de Lorgeril M, Hamazaki T. Kostucki W, et al. Is the use of cholesterol-lowering drugs for the prevention of cardiovascular complications in type 2 diabetics evidence-based? A systematic review. Rev Recent Clin Trials 2012;7:150-7

61. Anagnostis P, Athyros VG, Adamidou F. et al. Glucagon-like peptide-1-based therapies and cardiovascular disease: looking beyond glycaemic control. Diabetes Obes Metab 2011;13:302-12

62. Monami M, Iacomelli I, Marchionni N. et al. Dipeptidyl peptidase-4 inhibitors in type 2 diabetes: a meta-analysis of randomized clinical trials. Nutr Metab Cardiovasc Dis 2010;20:224-35

63. Williams-Herman D, Engel SS. Round E, et al. Safety and tolerability of sitagliptin in clinical studies: a pooled analysis of data from 10,246 patients with type 2 diabetes. BMC Endocr Disord 2010;10:7

- This pooled analysis of all Phase III studies with sitagliptin showed that the risk of cardiovascular events for sitagliptin versus comparators was reduced by $32 \%$ in patients with T2DM, an effect that remains to be confirmed in the ongoing TECOS trial.

64. Murohara T. Dipeptidyl peptidase-4 inhibitor: another player for cardiovascular protection. J Am Coll Cardiol 2012;59:277-9

65. (TECOS). Scos. TECOS: A Randomized, Placebo Controlled Clinical Trial to Evaluate Cardiovascular Outcomes After Treatment With Sitagliptin in Patients With Type 2 Diabetes Mellitus and Inadequate Glycemic Control. ClinicalTrials.gov Identifier. NCT00790205 Available from: http:/wwwclinicaltrialsgov/ct2/ show/NCT00790205? term=TECOS\&rank=1 published on line;doi

- TECOS is a long-term, event-driven, non-inferiority, cardiovascular outcomes trial evaluating the impact of sitagliptin when added to usual diabetes care in a large, high-risk population with T2DM, most of them receiving a statin (and presumably numerous on atorvastatin).

66. Gaede P, Vedel P, Larsen N, et al. Multifactorial intervention and cardiovascular disease in patients with type 2 diabetes. N Engl J Med 2003;348:383-93

67. Athyros VG, Tziomalos K. Karagiannis A, et al. Atorvastatin: safety and tolerability. Expert Opin Drug Saf 2010;9:667-74

68. Bernini F, Poli A, Paoletti R Safety of HMG-CoA reductase inhibitors: focus on atorvastatin. Cardiovasc Drugs Ther 2001;15:211-18

69. Waeber B, Buclin T, Grouzmann E. Angioedema during ACE and DPP-4 inhibition. Rev Med Suisse 2010;6:28-31

70. Engel SS, Williams-Herman DE, Golm GT, et al. Sitagliptin: review of preclinical and clinical data regarding incidence of pancreatitis. Int J Clin Pract 2010;64:984-90

71. Bhome R, Penn H. Rhabdomyolysis precipitated by a sitagliptin-atorvastatin drug interaction. Diabet Med 2012;29:693-4

72. Kao DP, Kohrt HE, Kugler J. Renal failure and rhabdomyolysis associated with sitagliptin and simvastatin use. Diabet Med 2008;25:1229-30

73. DiGregorio RV, Pasikhova Y. Rhabdomyolysis caused by a potential sitagliptin-lovastatin interaction. Pharmacotherapy 2009;29:352-6

74. Boucher BJ. Renal failure and rhabdomyolysis associated with sitagliptin and simvastatin use. But what about the amiodarone? Diabet Med 2009;26:192-3

75. Chan JC, Scott R, Arjona Ferreira JC. et al. Safety and efficacy of sitagliptin in patients with type 2 diabetes and chronic renal insufficiency. Diabetes Obes Metab 2008;10:545-55

76. Pendergrass M, Fenton C, Haffner SM. et al. Exenatide and sitagliptin are not associated with increased risk of acute renal failure: a retrospective claims analysis. Diabetes Obes Metab 2012, published on line 2012/01/25; doi: 10.HH/j.1463-1326.2012.01567.x. 